\title{
ON INTERPOLATING FUNCTIONS WITH MINIMAL NORM
}

\author{
A. STRAY AND K. O. ØYMA
}

\begin{abstract}
Let $H^{\infty}$ denote the Banach algebra of bounded analytic functions in the unit disc $\{z:|z|<1\}$. If $f$ is an extreme point in the unit ball of $H^{\infty}$, there is always a Blaschke product $B$, whose zeros form an interpolating sequence tending to one point of the unit circle, such that $\|f+B h\|_{\infty}>1$ if $h \in H^{\infty}$ and $h \neq 0$. An application of this result to the theory of best approximation is given.
\end{abstract}

Let $H^{\infty}$ denote the Banach algebra of all bounded analytic functions in the unit disc $D$. A sequence $S=\left\{z_{\nu}\right\} \subset D$ is the zero set of a nonzero function in $H^{\infty}$ if and only if $\Sigma_{\nu} 1-\left|z_{\nu}\right|<\infty$. The Blaschke product corresponding to $S$ is given by

$$
B(z)=\prod_{\nu} \frac{\left|z_{\nu}\right|}{-z_{\nu}} \frac{z-z_{\nu}}{1-\bar{z}_{\nu} z}
$$

if $z_{\nu} \neq 0, \nu=1,2, \ldots$ It is well known that $B \in H^{\infty}$ and that $B$ has norm one and $S=B^{-1}(0)$. We also remark that $B$ is analytic at each $z_{0} \in T=$ $\{z:|z|=1\}$ not in the closure of $\left\{\dot{z}_{v}\right\}$.

If the restriction map $f \rightarrow\left\{f\left(z_{\nu}\right)\right\}_{\nu}$, maps $H^{\infty}$ onto $l^{\infty}, S$ is called an interpolating sequence. For more details about $H^{\infty}$ and interpolating sequences, we refer to [4].

In this note we prove the following result.

THEOREM 1. If $h$ is an extreme point in the unit ball of $H^{\infty}$, there exists an interpolating sequence $\left\{z_{\nu}\right\}$ tending to one point, such that $\|f\|>\|h\|$ if $f \in H^{\infty}, f \neq h$, and $f\left(z_{\nu}\right)=h\left(z_{\nu}\right), \nu=1,2, \ldots$

REMARK. This result, which extends recent work announced by D. S. Jerison [5], has also been proved independently by D. Marshall using similar ideas.

There is a consequence in the theory of best approximation by $H^{\infty}$ functions which we would like to point out before giving the proof of Theorem 1.

Let $z_{0}$ denote the clusterpoint of $\left\{z_{\nu}\right\}$ on $T$. We assume that $h$ does not extend continuously to any point on $T$. Let $f_{1} \in H^{\infty}$ have analytic continuation across $T \backslash\left\{z_{0}\right\}$ and satisfy $f_{1}\left(z_{\nu}\right)=h\left(z_{\nu}\right), \nu=1,2, \ldots$ Such a function exists [3].

Received by the editors January 19, 1977.

AMS (MOS) subject classifications (1970). Primary 30A80, 41A50, 46J15.

Key words and phrases. Minimal interpolating function, best approximation. 
If $B$ denotes the Blaschke product corresponding to $\left\{z_{v}\right\}$, we can write $h=f_{1}+B f$, where $f \in H^{\infty}$ is the unique best $H^{\infty}$-approximation to $f_{1} / B$ on $T$. (If $g \in H^{\infty}$, we assume it is extended to $T$ by taking the radial limits $g^{*}\left(e^{i \theta}\right)=\lim _{r \rightarrow 1} g\left(r e^{i \theta}\right)$.) So even if $f_{1} / B$ extends to be analytic near $T \backslash\left\{z_{0}\right\}$, $f$ may be discontinuous at each point of $T \backslash\left\{z_{0}\right\}$. This should be compared with recent work of $L$. Carleson and S. Jacobs [1].

Proof OF THEOREM 1 . If $g \in H^{\infty}, g^{*}$ will always denote its radial limits on $T$, and $\left\|g^{*}\right\|_{\infty}$ denotes the norm of $g^{*}$ in $L^{\infty}(d \theta)$, where $d \theta$ denotes Lebesgue measure on $T$. It is well known [4] that $g$ is an extreme point in $H^{\infty}$ if and only if

$$
\frac{1}{2 \pi} \int_{-\pi}^{\pi} \log \left(1-\left|g^{*}\right|\right) d \theta=-\infty
$$

Since $h$ is extreme, there is a number $\theta_{0}$ such that $\int_{\theta_{0}-t}^{\theta_{0}+t} \log \left(1-\left|h^{*}\right|\right) d \theta=$ $-\infty$ for any $t>0$. We may assume $\theta_{0}=0$. The idea of the proof is as follows: We choose an interpolating sequence $\left\{z_{v}\right\}$ tending to 1 such that $\left|h\left(z_{v}\right)\right| \rightarrow 1$ and with the following property:

If $f \in K=\left\{g \in H^{\infty}:\|g\|=1, g\left(z_{\nu}\right)=h\left(z_{\nu}\right), \nu=1,2, \ldots\right\}$, then $f$ is an extreme point in the unit ball of $H^{\infty}$. Since $h \in K$ and $K$ is convex, this implies that $K=\{h\}$.

To find $\left\{z_{\nu}\right\}$, let $I_{n}=\left\{z \in T: 2(n+1)^{-1}<|z-1| \leqslant 2 n^{-1}\right\}$ for $n=$ $1,2, \ldots$ Assume first there are constants $M_{n}$ such that $\left|h^{*}\right| \leqslant M_{n}<1$ on $I_{n}$, $n=1,2, \ldots$ We choose compact sets $K_{n} \subset I_{n}, n=1,2, \ldots$, with the following two properties for each $n$ :

$$
\begin{gathered}
\int_{K_{n}} \log \left(1-\left|h^{*}\right|\right) d \theta \leqslant 2^{-1} \int_{I_{n}} \log \left(1-\left|h^{*}\right|\right) d \theta ; \\
\lim _{r \rightarrow 1} f_{r}\left(e^{i \theta}\right)=f^{*}\left(e^{i \theta}\right) \text { exists uniformly for } e^{i \theta} \in K_{n} .
\end{gathered}
$$

Fix $n$, and subdivide $I_{n}$ into a collection of $\operatorname{arcs}\left\{I_{n, k}\right\}_{k}$ of equal length $\delta_{n}$ which is so small that

$$
\left|h^{*}\left(e^{i \theta}\right)-h\left(r e^{i \theta}\right)\right|<1-M_{n}
$$

if $e^{i \theta} \in K_{n}$ and $r \geqslant 1-2 \delta_{n}$.

From $\left\{I_{n, k}\right\}_{k}$ we choose a subcollection $\left\{J_{n, k}\right\}_{k}$ such that $J_{n, k} \cap K_{n} \neq \varnothing$ for each $k$, and with the following two properties:

$$
\sum_{k} \int_{K_{n} \cap J_{n, k}} \log \left(1-\left|h^{*}\right|\right) d \theta \leqslant 4^{-1} \int_{K_{n}} \log \left(1-\left|h^{*}\right|\right) d \theta .
$$

(ii) No arc of length less than $2 \delta_{n}$ on $T$ can intersect to different arcs from $\left\{J_{n, k}\right\}_{k}$.

Let ess $\sup \left\{\left|h^{*}\left(e^{i \theta}\right)\right|, e^{i \theta} \in K_{n} \cap J_{n, k}\right\}=1-\eta_{n k}$. Then we get from (4), and since $1-\eta_{n k} \leqslant M_{n}$, that there is a point $\zeta_{n k} \in J_{n, k}$ such that if $z_{n k}=(1$ $\left.-2 \delta_{n}\right) \zeta_{n k}$, then

$$
\left|h\left(z_{n k}\right)\right| \geqslant 1-2 \eta_{n k}-\left(1-M_{n}\right) \geqslant 1-3 \eta_{n k} .
$$


Let $\tilde{J}_{n, k}$ be the arc centered at $\zeta_{n, k}$ and with length $2 \delta_{n}$. By (ii), $\left\{\tilde{J}_{n, k}\right\}_{k}$ is a disjoint collection of arcs. If $S_{n}=\left\{z_{n, k}\right\}_{k}$, we define our sequence $\left\{z_{v}\right\}_{\nu}$ as the union of all $S_{n}, n=1,2, \ldots$.

We now make the following claim:

If $f \in H^{\infty},\|f\| \leqslant 1$, and $\left|f\left(z_{\nu}\right)\right| \geqslant\left|h\left(z_{\nu}\right)\right|, \nu=1,2, \ldots$, then $\int_{-\pi}^{\pi} \log (1$ $\left.-\left|f^{*}\right|\right) d \theta=-\infty$.

To prove the claim, we use that if $\left|f\left(z_{n, k}\right)\right| \geqslant\left|h\left(z_{n k}\right)\right| \geqslant 1-3 \eta_{n k}$, then there is a set $E_{n k} \subset \tilde{J}_{n, k}$ and absolute constants $A_{1}$ and $A_{2}$ such that

$$
\left|f^{*}\right| \geqslant 1-A_{1} \eta_{n k} \text { on } E_{n k}
$$

and

$$
\text { length } \begin{aligned}
E_{n k} & \geqslant A_{2} \text { length } \tilde{J}_{n, k}=2 A_{2} \delta_{n} \\
& =2 A_{2} \text { length } J_{n, k} .
\end{aligned}
$$

The existence of $A_{1}$ and $A_{2}$ comes out of estimating the Poisson kernel for $z_{n, k}$ on the $\operatorname{arc} \tilde{J}_{n, k}$.

Using (5), (6) and the definition of $\eta_{n, k}$, it is easy to see that

$$
\begin{aligned}
& \int_{E_{n k}} \log \left(1-\left|f^{*}\right|\right) d \theta \\
& \quad \leqslant 2 A_{2} \int_{K_{n} \cap J_{n, k}}\left(\log \left(1-h\left|h^{*}\right|\right)+\log A_{1}\right) d \theta .
\end{aligned}
$$

If we sum these inequalities for each $J_{n, k} \in\left\{J_{n, k}\right\}_{k}$, and then for $n=$ $1,2, \ldots$, we get, using property (ii) about $\left\{J_{n, k}\right\}_{k}$ and (2), that

$$
\begin{aligned}
\int_{T} \log \left(1-\left|f^{*}\right|\right) d \theta & \leqslant \sum_{n} \sum_{k} \int_{E_{n k}}\left(\log 1-\left|f^{*}\right|\right) d \theta \\
& \leqslant 4^{-1} A_{2} \int_{T}\left(\log 1-\left|h^{*}\right|+\log A_{1}\right) d \theta=-\infty,
\end{aligned}
$$

which proves the claim.

We verify now that $\left\{z_{v}\right\}_{\nu}$ is an interpolating sequence. By a characterization of such sequences essentially due to L. Carleson [2, Lemma 1], we need only verify the following two inequalities:

$$
\sum_{z_{\nu} \in R(\theta, h)}\left(1-\left|z_{\nu}\right|\right) \leqslant C \cdot h
$$

(where $R(\theta, h)=\{z: \theta \leqslant \arg z \leqslant \theta+h, 0<1-|z| \leqslant h\}$ and $C$ is independent of $h)$, and

$$
\inf _{\mu \neq \nu}\left|\frac{z_{\nu}-z_{\mu}}{1-\bar{z}_{\mu} z_{\nu}}\right| \geqslant \delta
$$

for some $\delta>0$ and $\nu=1,2, \ldots$.

By construction there is no $z_{\mu}$ with $\mu \neq \nu$ in the sector

$$
S_{\nu}=\left\{z: \arg z_{\nu}-\frac{1}{2}\left(1-\left|z_{\nu}\right|\right)<\arg z<\arg z_{\nu}+\frac{1}{2}\left(1-\left|z_{\nu}\right|\right)\right\} .
$$


It is easy to verify that this fact implies both (a) and (b) above. In fact, the arc $\tilde{J}_{\nu}=T \cap \partial S_{\nu}$, corresponding to $z_{\nu}$, has length $1-\left|z_{\nu}\right|$, and since the collection $\left\{J_{\nu}\right\}_{\nu}$ is disjoint, (a) must hold with $c=2$. Elementary geometric considerations also show that the disc $D_{\nu}=\left\{z:\left|\left(z-z_{\nu}\right) /\left(1-z_{\nu} z\right)\right|<\delta\right\}$ is contained in $S_{\nu}$ if $\delta$ is sufficiently small, but independent of $\nu$.

The proof is complete except that we assumed $\left|h^{*}\right| \leqslant M_{n}<1$ on each $I_{n}$. If this is not the case, we divide $h$ by a suitable outer function $F$ such that $h_{1}=h \cdot F^{-1}$ is an extreme point satisfying all our hypothesis.

We can arrange it so that $|F(z)|>1, z \in D$. If the above claim is proved for $h_{1}$, it is certainly also true for $h$.

\section{REFERENCES}

1. L. Carleson and S. Jacobs, Best uniform approximation by analytic functions, Ark. Mat. 10 (1972), 219-229.

2. J. Garnett, Interpolating sequences for bounded harmonic functions, Indiana Univ. Math. J. 21 (1972), 187-192.

3. E. A. Heard and J. H. Wells, An interpolation problem for subalgebras of $H^{\infty}$, Pacific J. Math. 28 (1969), 543-553.

4. K. Hoffman, Banach spaces of analytic functions, Prentice-Hall, Englewood Cliffs, N.J., 1962.

5. D. S. Jerison, Sur un théorème d'interpolation de R. Nevanlinna, C. R. Acad. Sci. Paris Ser. A (1976), 1291-1293.

Agder Distriktshogskole, Postboks 607, N-4601 Kristiansand, Norway 